\title{
Clinical Characteristics of Induced Nonclinical Ventricular Tachycardia in Nonischemic Cardiomyopathy
}

\author{
Katsunori OKAJIMA, ${ }^{1} \mathrm{MD}$, Akihiro YoshIDA, ${ }^{1} \mathrm{MD}$, Yoshio OHNISHI, ${ }^{1} \mathrm{MD}$, \\ and Mitsuhiro YOKOYAMA, ${ }^{1} \mathrm{MD}$
}

\begin{abstract}
SUMMARY
The clinical significance of induced nonclinical ventricular tachycardia (NCVT) in nonischemic dilated cardiomyopathy (DCM) remains controversial. Twenty-eight patients with sustained VT or ventricular fibrillation related to DCM underwent programmed ventricular stimulation (PVS) to induce VT. However, VT was not induced in four patients. Based on the morphology of induced ventricular arrhythmia, we classified the remaining 24 patients into NCVT $(n=11)$ and clinical VT (CVT) groups $(n=13)$, then evaluated the prognosis for a mean follow-up period of 22 months. The cycle length of induced NCVT was significantly shorter than that of induced CVT $(277 \pm 38 \mathrm{~ms}$ vs $325 \pm 63 \mathrm{~ms}, P<0.05)$. Appropriate antiarrhythmic agents were selected by serial PVS in $36 \%$ of the NCVT group and in $38 \%$ of the CVT group (4/11 vs 5/13). Among patients who had been treated by PVS guided drug therapy, arrhythmic events were observed in $75 \%$ of the NCVT group and $80 \%$ of the CVT group (3/4 vs $4 / 5$ ). The total incidence of sudden death in the NCVT group was higher than that in the CVT group (5/11: $45 \%$ vs 4/13: $31 \%$ ). In conclusion, induced NCVT and CVT are refractory to pharmacological therapy and both have an important characteristic value in DCM. (Jpn Heart J 2002; 43: 643-654)
\end{abstract}

Key words: Nonischemic dilated cardiomyopathy, Programmed ventricular stimulation, Nonclinical ventricular tachycardia

PATIENTS with nonischemic dilated cardiomyopathy (DCM) are at a high risk of sudden death, which may be associated with ventricular tachyarrhythmias. ${ }^{1-4)}$ Electrophysiological studies (EPS) have identified antiarrhythmic agents that prolong the time to a recurrence of arrhythmia in patients with sustained ventricular tachycardia (VT) or ventricular fibrillation (VF). ${ }^{5}$ This approach to the treatment of VT is based on the premise that programmed ventricular stimulation (PVS) in the laboratory reliably reproduces electrocardiographically documented, spontaneous clinical VT. However, the relationship between the characteristics of induced and spontaneous VT in the same patient is not always

From ${ }^{1}$ Division of Cardiovascular and Respiratory Medicine, Kobe University Graduate School of Medicine, Hyogo, Japan. Address for correspondence: Akihiro Yoshida, MD, 7-5-2, Kusunoki-cho, Chuo-ku, Kobe, Hyogo Japan.

Received for publication January 11, 2002.

Revised and accepted June 6, 2002. 
consistent. ${ }^{\text {() }}$ Constantin, et al reported that antiarrhythmic agents frequently rendered sustained VT non-inducible or non-sustained and suppression of inducible sustained VT by the effective antiarrhythmic drug predicts the long-term prevention of spontaneous recurrences in nonischemic dilated cardiomyopathy. ${ }^{7)}$ However, the clinical significance of inducible nonclinical ventricular tachyarrhythmias (not documented during spontaneous episodes) in DCM has not been published. The purposes of this study were to determine whether or not inducible nonclinical VT (NCVT) in these patients has a clinical role, and to clarify the effectiveness of amiodarone in treating NCVT.

\section{Methods}

Patients: The study population comprised 28 patients, including 18 men and 10 women aged 38 to 65 years (mean $54 \pm 10$ years). Patients with nonischemic organic heart disease were admitted to the Hospital of Kobe University School of Medicine or Himeji Cardiovascular Center for evaluation of documented sustained monomorphic VT (23 patients), aborted cardiac sudden death (2 patients), incessant non-sustained VT (1 patient) or unknown syncope (2 patients). Clinical hemodynamic, echocardiographic, and angiographic findings revealed that nonischemic organic heart disease was due to idiopathic dilated cardiomyopathy in 17 patients, cardiac sarcoidosis in 5, chronic myocarditis in 2, aortic valve regurgiment in 2, ventricular septal defect in 1, and traumatic left ventricular aneurysm in 1 . We excluded patients with coronary artery disease demonstrated by documented myocardial infarction or the cineangiographic presence of $75 \%$ or greater stenosis of one or more of the coronary arteries ${ }^{8)}$ and those with arrhythmogenic right ventricular cardiomyopathy.

Signal-averaged electrogram (SAECG): Orthogonal bipolar X, Y, and Z leads were recorded until a noise level of $0.4 \mu \mathrm{V}$ was reached using standard procedures (Fukuda Denshi FDX-6521, Tokyo). The record signal was digitized, and the resultant data underwent signal averaging and filtering using a bandpass filter with a range of 40 to $250 \mathrm{~Hz}$. The QRS duration, root mean square voltage of the terminal $40 \mathrm{~ms}$ of the filtered QRS complex (RMS40), and the duration of the low amplitude $(<40 \mathrm{~Hz})$ signals of the terminal filtered QRS complex (LAS40) were calculated using an automated algorithm. In patients with a normal QRS complex (QRS $\leq 110 \mathrm{~ms}$ ), the SAECG determined by time-domain analysis was considered positive if the filtered QRS duration was $>114 \mathrm{~ms}$ and RMS 40 was $<20 \mu \mathrm{V}$ or LAS 40 was $>38 \mathrm{~ms}$ at $40-\mathrm{Hz}$ filtering. In patients with a wide QRS complex (QRS $>110 \mathrm{~ms}$ ), the SAECG was considered positive if two or three of the following criteria were positive: filtered QRS duration of $>145 \mathrm{~ms}$, RMS 40 of $<17$ $\mu \mathrm{V}$, and LAS 40 of $>45 \mathrm{~ms}$ at $40-\mathrm{Hz}$ filtering. ${ }^{9-11)}$ 
Electrophysiological study: EPS was performed on non-sedated, post-absorptive patients after obtaining their written informed consent. Initial EPS was performed after antiarrhythmic agents had been discontinued for at least five half-lives. Three 6F quadripolar electrode catheters were usually inserted from the right femoral vein under fluoroscopic guidance to the high right atrium, the His-bundle area, and the right ventricular apex and/or outflow tract. The PVS protocol consisted of single, double, and triple extra stimuli during ventricular paced cycle lengths of $600 \mathrm{~ms}$ and $400 \mathrm{~ms}$, and rapid ventricular pacing via a programmable stimulator (Cardiac Stimulator SEC-3102, Nihon Kohden, Tokyo). Stimuli were delivered as rectangular $1 \mathrm{~ms}$ pulses at two to four times the diastolic threshold. The stimulation was initially applied at the right ventricular apex, then at the right ventricular outflow tract up to double extra stimuli. Rapid pacing was performed until 2:1 ventricular capture occurred, and finally triple extra stimuli were used from each site at basic cycle lengths of $600 \mathrm{~ms}$ and $400 \mathrm{~ms}$. The stimulation was continued until sustained VT or VF, which had been terminated by direct current shock because of hemodynamic collapse, was induced. If the induced VT was hemodynamically tolerated, the following stimulations would proceed until the VT as above was induced or the protocol was completed. During this protocol, all 12 standard ECG leads were simultaneously recorded with the intracardiac ECGs.

Definitions: Sustained VT: Ventricular tachycardia lasting more than 30 seconds or requiring termination because of hemodynamic collapse. Non-sustained VT: Ventricular tachycardia lasting more than 6 beats with rates above 100 beats/min that terminated spontaneously within 30 seconds. clinical VT (CVT): Inducible VT that matched the morphology of the patient's documented, spontaneous monomorphic VT. ${ }^{12)}$ Nonclinical VT (NCVT): Inducible monomorphic or polymorphic VT that was not known to have spontaneously arisen. Non-inducibility: Less than 5 repetitive ventricular responses were induced until the PVS was completed. Sudden death: Death within one-half hour of symptoms in a patient free of symptoms during the preceding 24 hours and in whom no other cause of death was discernible. ${ }^{13)}$

Antiarrhythmic medication: The choice of therapy was based on the results of EPS. Antiarrhythmic agents were defined as effective if fewer than 5 repetitive ventricular responses were induced throughout the complete PVS. The maximally tolerated dose of class I antiarrhythmic agents was intravenously administered to 20 patients with inducible VT, and the same PVS protocols were repeated. Amiodarone was administered to 4 patients at a loading dose of $400 \mathrm{mg}$ per day for 2 weeks, followed by a maintenance dose of $200 \mathrm{mg}$ per day for more than 1 week. The same PVS protocols were repeated after an average interval of amiodarone administration of $25 \pm 8$ days. If an effective antiarrhythmic agent 
could not be determined for the patients with inducible VT based on the results of EPS, the choice of therapy was based on the results of Holter monitoring or empirical decision.

Follow up: VT was not induced in 4 patients. Based on the morphology of induced arrhythmia, we classified the remaining 24 patients into nonclinical VT (NCVT group; $n=11$ ) and clinical VT groups (CVT group; $n=13$ ). When patients had both types of inducible VT, they were classified as NCVT. The patients were followed up through repeat visits to the arrhythmia research clinic in the Hospital of Kobe University School of Medicine or Himeji Cardiovascular Center every two to four weeks for a mean period of $21 \pm 23$ months. An arrhythmic event was defined as a recurrence of ventricular tachyarrhythmia, the first appropriate shock delivery of an implantable cardioverter defibrillator (ICD), or sudden death, including aborted sudden death. When an out-of-hospital death occurred, we attempted to identify the factors surrounding the event to identify the cause.

Statistical Analysis: Data are expressed as means \pm SD. Comparisons between groups were performed using the unpaired Student's $t$-test or the chi-square method. ${ }^{14)}$ Survival free curves of arrhythmic events were constructed using the Kaplan-Meier method, and the log-rank statistic was used to determine survival differences.

\section{Results}

Patient characteristics: The NCVT group consisted of 11 patients, including 5 with both inducible types of VT. The CVT group consisted of 13 patients. Their characteristics are summarized in Table I. Mean age, sex, incidence of atrial

Table I. Clinical Characteristics of the 24 Study Patients

\begin{tabular}{lccc}
\hline & $\begin{array}{c}\text { NCVT group } \\
(n=11)\end{array}$ & $\begin{array}{c}\text { CVT group } \\
(n=13)\end{array}$ & $P$ value \\
\hline Age (Years) & $56 \pm 9$ & $53 \pm 10$ & $\mathrm{NS}$ \\
Gender (male: female) & $8: 3$ & $6: 7$ & $\mathrm{NS}$ \\
Atrial fibrillation (+) & $2 / 11$ & $3 / 13$ & $\mathrm{NS}$ \\
Structural heart disease & & & \\
$\quad$ ldiopathic dilated cardiomyopathy & 6 & 8 & \\
$\quad$ Cardiac sarcoidosis & 1 & 4 & \\
$\quad$ Others & 4 & 1 & $\mathrm{NS}$ \\
LV function & $116 \pm 16$ & $137 \pm 50$ & $\mathrm{NS}$ \\
$\quad$ LVEDVI (ml/m $\left.{ }^{2}\right)$ & $43 \pm 13$ & $34 \pm 13$ & $\mathrm{NS}$ \\
$\quad$ Ejection fraction (\%) & $71 \%$ & $90 \%$ & $\mathrm{NS}$ \\
Holter ECG (incidence of VT) & $86 \%$ & $63 \%$ & $\mathrm{NS}$ \\
SAECG (+) & $19 \pm 19$ & $24 \pm 28$ & \\
Follow-up Period (mo) & & & \\
\hline
\end{tabular}

LVEDVI=left ventricular end-diastolic volume index, SAECG=signal-averaged electrocardiogram, NS=statistically not significant. 
fibrillation, left ventricular end diastolic volume index, left ventricular ejection fraction, and incidence of VT by Holter ECG did not significantly differ between the two groups. The SAECG was performed in 7 NCVT patients and in 8 CVT patients. The SAECG in the remaining patients could not be evaluated because of atrial fibrillation or ventricular pacing rhythm. Six patients $(86 \%)$ in the NCVT group and 5 patients $(63 \%)$ in the CVT group fulfilled the criteria for positive SAECG. Beta-blockade was administered to 5 NCVT patients and 6 CVT patients.

Characteristics of spontaneous and induced ventricular tachyarrhythmias: An example of induced nonclinical VT is shown in Figure 1. Electrophysiological characteristics are summarized in Table II. In the NCVT group, the morphology of spontaneous VTs showed right bundle branch block (RBBB) in 6 patients and left bundle branch block (LBBB) in 5 patients. Ventricular tachyarrhythmias were induced by PVS in 10 patients and by isoproterenol alone in 1 . Fifty percent of the patients in this group needed triple extra stimuli for VT induction. On the other hand, 16 VTs were spontaneously detected in 13 patients in the CVT group. Ten of these were the RBBB type and 6 were the LBBB type. VTs were induced by PVS in all patients. Fifteen percent of patients in this group needed triple extra stimuli for VT induction. Five VTs showed RBBB type with superior axis in both groups, but idiopathic VT occurring from the left posterior fascicle was not detected in any VTs. Although the cycle lengths of spontaneous VT did not significantly differ between the two groups, the cycle lengths of induced VT in the NCVT group were significantly shorter than those in the CVT group (272 $\pm 40 \mathrm{~ms}$ vs $327 \pm 64 \mathrm{~ms}, P<0.05)$. Induction of NCVT had required a more aggressive approach, so triple extra stimuli may explain this difference.

Table II. Electrophysiological Characteristics

\begin{tabular}{lccc}
\hline & $\begin{array}{c}\text { NCVT group } \\
(n=11)\end{array}$ & $\begin{array}{c}\text { CVT group } \\
(n=13)\end{array}$ & $P$ value \\
\hline Spontaneous VT & & & \\
Cycle length (ms) & $321 \pm 67$ & $345 \pm 64$ & $\mathrm{NS}$ \\
Morphology (RBBB/LBBB) & $6 / 5$ & $10 / 6$ & $\mathrm{NS}$ \\
Induced VT & & & \\
lnduction site (RVA/RVOT/LV) & $4 / 5 / 1$ & $9 / 4 / 0$ & \\
lnduction mode (T/D/S/B) & $5 / 3 / 0 / 2$ & $2 / 5 / 2 / 4$ & \\
Morphology (RBBB/LBBB/PMVT) & $4 / 6 / 4$ & $8 / 5$ & $\mathrm{NS}$ \\
T/ (T+D+S+B) & $50 \%$ & $15 \%$ & \\
Cycle length (ms) & $272 \pm 40$ & $327 \pm 64$ & $<0.05$ \\
\hline
\end{tabular}

$\mathrm{VT}=$ ventricular tachycardia; Morphology=morphology of VT confirmed by 12 lead ECG; RBBB=right bundle branch block type; LBBB=left bundle branch block type; PMVT=polymorphic VT; RVA=right ventricular apex; RVOT=right ventricular outflow tract; $\mathrm{LVA}=$ left ventricular apex; T,D, and $\mathrm{S}=$ triple, double, and single extra stimuli; $\mathrm{B}=$ burst pacing. 


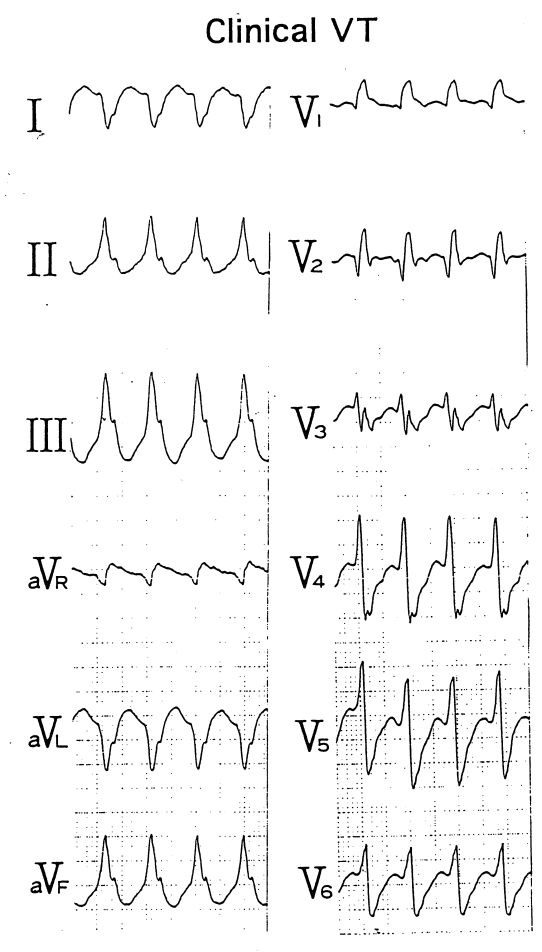

Induced non-clinical VT

\section{I}
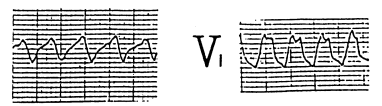

II
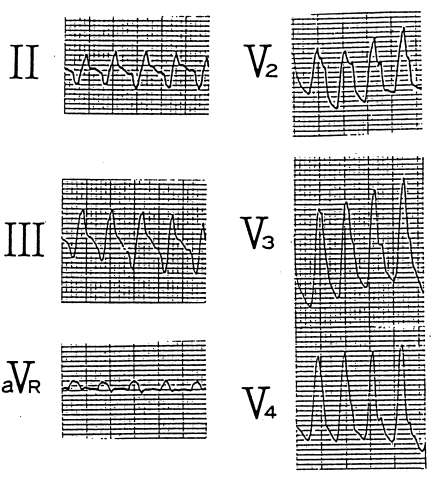

$\mathrm{aV}_{\mathrm{R}}$

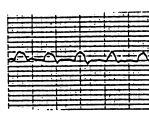

$\mathrm{V}_{4}$
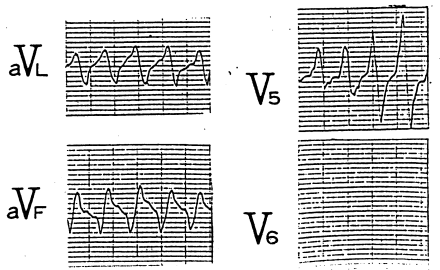

Figure 1. A representative case of induced nonclinical VT. Cycle length of induced nonclinical VT was shorter than spontaneous clinical VT (260 vs $380 \mathrm{~ms}$ ). V6 lead of right panel was not successfully recorded.

Serial drug testing: Twenty-four patients with inducible VT underwent a total of 34 trials of different drug regimens (mean of 1.4 trials per patient). The following agents were used for serial testing: procainamide, flecainide, disopyramide, aprindine, mexiletine, lidocaine, or amiodarone. In the NCVT group, class I antiarrhythmic agents rendered the arrhythmia noninducible in 3 of 9 patients and amiodarone rendered the arrhythmia noninducible in 1 of 2 patients. In total, the effective antiarrhythmic agents could be determined in 4 patients $(36 \%)$ based on the EPS. On the other hand, in the CVT group, class I antiarrhythmic agents rendered the arrhythmia noninducible in 5 of 11 patients while amiodarone did not render the arrhythmia noninducible in 2 patients. The effective antiarrhythmic agents based on the EPS could be determined in a total of 5 patients. A total of 9 patients were discharged on a regimen of the antiarrhythmic agent that had rendered VT noninducible. The remaining 7 patients in the NCVT group and 8 patients in the CVT group were administered the antiarrhythmic agents based on the results of Holter monitoring or empirical decision.

Follow-up of the nonclinical VT group: In the NCVT group, arrhythmic events 
occurred in 3 of 4 patients (75\%) on EPS guided antiarrhythmic agents during a mean follow-up period of $19 \pm 19$ months. Sustained VT recurred in 1 patient and the morphology of VT detected by 12-lead ECG was different from the previously documented VT. Sudden death and aborted sudden death occurred in 2 other patients. All 3 of these patients were administered class I antiarrhythmic agents and the remaining 1 patient without a recurring arrhythmic event was administered amiodarone. The two patients, except the sudden death patient, had been implanted with an ICD after the arrhythmic events.

Among the remaining 7 patients in whom EPS guided antiarrhythmic agents could not be determined, 3 (43\%) arrhythmic events occurred. One patient administered a class I antiarrhythmic agent died suddenly, and the other 2 patients (1 patient: class I antiarrhythmic agent, 1 patient: amiodarone) received appropriate ICD delivery (Figure 2). In one recurrent VT, the morphology could be determined by 12-lead ECG and it was different from the previously documented VT. In the remaining 4 patients without VT recurrence, 3 patients were administered amiodarone and 1 patient was administered a class I antiarrhythmic agent.

Follow-up of the clinical VT group: In the CVT group, arrhythmic events occurred in 4 of 5 patients $(80 \%)$ on EPS guided class I antiarrhythmic agents during a mean follow-up period of $24 \pm 28$ months. Three patients experienced VT recurrences and 1 patient died suddenly. The morphology of the recurrent VT was unknown because they could not be recorded by 12-lead ECG. One was

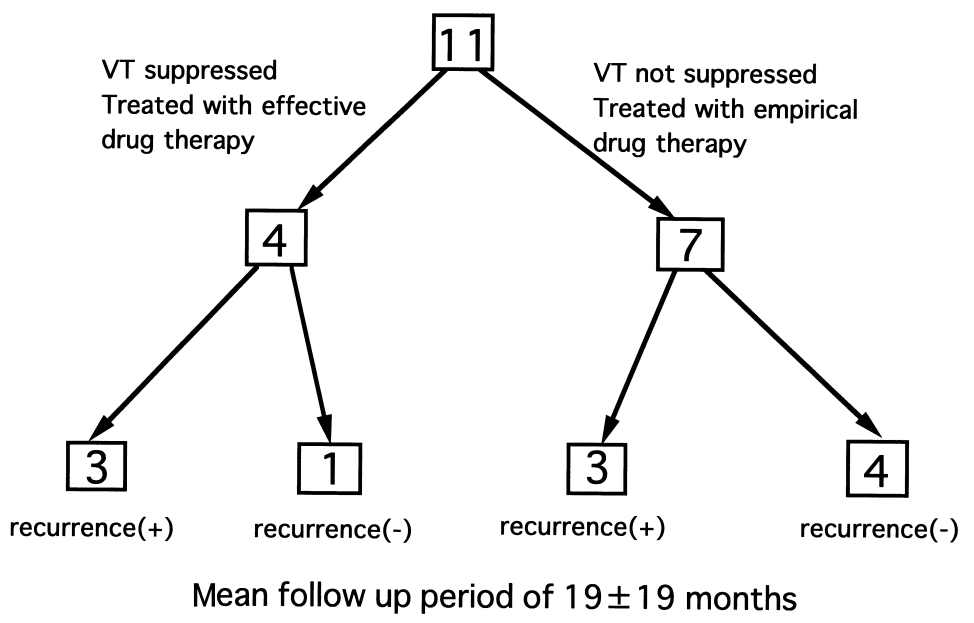

Figure 2. Relationship of results of electrophysiological study to outcome in the nonclinical VT (NCVT) group of 11 patients. $\mathrm{SCD}=$ sudden cardiac death. 
implanted with an ICD after the arrhythmic event. Among the remaining 8 patients in whom EPS guided antiarrhythmic agents could not be determined, 3 (38\%) arrhythmic events occurred. One patient with amiodarone died suddenly, and the other 2 patients ( 1 patient with amiodarone and another with class I antiarrhythmic agent) had VT recurrences. One of the 2 patients was implanted with an ICD after the VT recurrence (Figure 3). In the remaining 5 patients without recurrent arrhythmic events, 2 patients were administered amiodarone and 3 patients were administered class I antiarrhythmic agents.

Comparison of follow-up between NCVT and CVT groups: In total, arrhythmic events were observed in 6 patients in the NCVT group and in 7 patients in the CVT group (55\% vs 54\%). The cumulative incidences of any arrhythmic events were similar in both groups during the follow-up period (Figure 4A). Among these patients, sudden death (including aborted sudden death or appropriate ICD delivery) occurred in 5 patients in the NCVT group and 3 patients in the CVT group (45\% vs 23\%). Even among the patients with EPS guided effective antiarrhythmic agents, arrhythmic events occurred in 75\% of the NCVT group and in $80 \%$ of the CVT group ( 3 of 4 patients vs 4 of 5 patients), and sudden death occurred in $50 \%$ and $20 \%$ ( 2 of 4 patients vs 1 of 5 patients), respectively (Figure 4B).

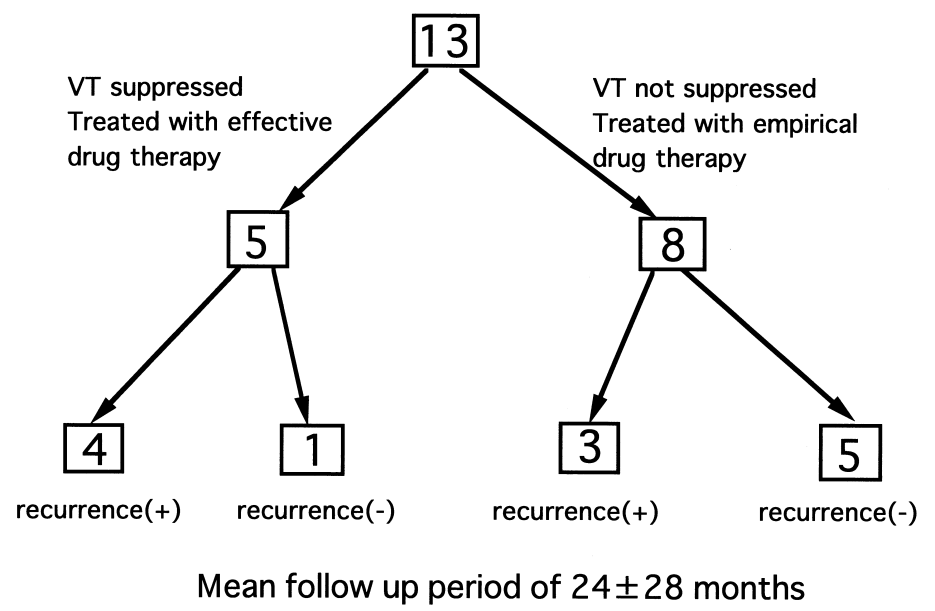

Figure 3. Relationship of results of electrophysiological study to outcome in the clinical VT (CVT) group of 13 patients. 

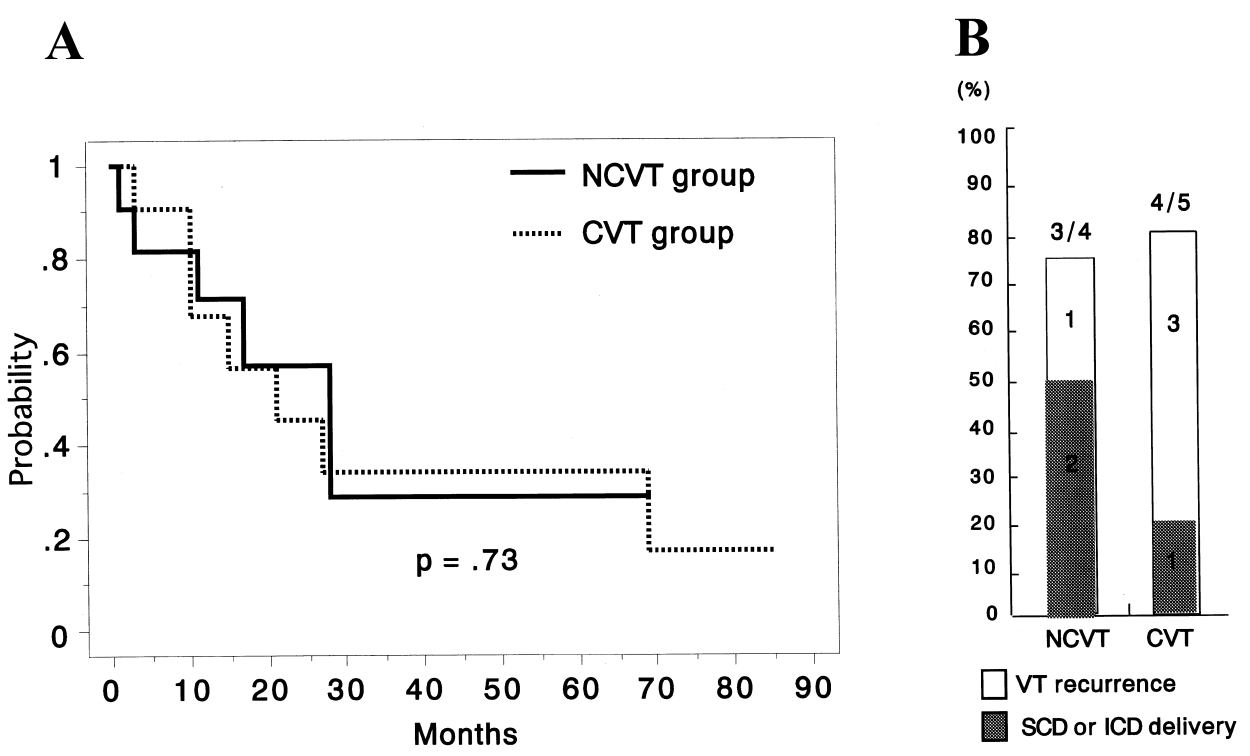

Figure 4. A: Kaplan-Meier survival curves depicting freedom from arrhythmic events in the NCVT group (solid line) compared to the CVT group (dotted line). B: The rate of arrhythmic events and sudden cardiac death or appropriate ICD delivery in the patients who were treated with effective antiarrhythmic agents.

\section{Discussion}

Major findings: The key finding of the present study is that inducible NCVT, like clinical VT, is refractory to pharmacological therapy in DCM. All patients who were evaluated in this study had life-threatening ventricular tachyarrhythmias related to DCM. Patient characteristics including parameters such as mean age, sex, left ventricular function, the number of patients taking beta-blockers, incidence of VT in Holter ECG, and SAECG did not differ significantly between the NCVT and CVT groups. In contrast, one electrophysiological feature differed significantly: the cycle lengths of inducible NCVT were shorter than those of CVT. This observation is similar to the findings reported by Michael, et al ${ }^{15}$ and Fred, et al. ${ }^{16)}$ The shorter cycle lengths of inducible NCVT may be consistent with the finding that NCVT induction required a more aggressive approach, namely triple the amount of extrastimuli (50\% of NCVT vs $17 \%$ of CVT). ${ }^{17}$ )

Outcome based on results of electrophysiological study: The role of EPS in the management of patients with symptomatic ventricular tachyarrhythmias and ischemic heart disease is established. The results of EPS-guided antiarrhythmic therapy have a high predictive value. ${ }^{18,19)}$ Some reports indicate that antiarrhythmic agents frequently render sustained VT noninducible or nonsustained and that 
antiarrhythmic drug suppression of inducible sustained VT predicts the long-term prevention of spontaneous recurrences. ${ }^{720,21)}$ However, other reports suggest that EPS is of limited value for arrhythmia risk evaluation in idiopathic dilated cardiomyopathy. ${ }^{22,23)}$ Milner, et al found no difference in arrhythmia recurrence between patients with and without inducible ventricular tachyarrhythmias in an initial study. Furthermore, arrhythmia suppression during serial drug testing did not predict outcome; arrhythmia recurred in 5 of 9 patients in whom arrhythmias had been suppressed. ${ }^{23)}$ The present study showed inducible NCVT predicted a poor outcome for patients with DCM and that the morphology of the recurrent VT were different from the previous ones, suggesting that many kinds of arrhythmic substrate for lethal arrhythmia might exist in patients with inducible NCVT. The recurrence rate of $75 \%$ and the cardiac sudden death (or appropriate ICD delivery) rate of $50 \%$ among patients in whom effective antiarrhythmic agents were identified based on EPS suggests that EPS is of limited value to guide drug therapy.

Catheter ablation: Rothman, et al have described the importance of inducible nonclinical tachyarrhythmias in patients with myocardial infarction. ${ }^{12)}$ They showed that radiofrequency catheter ablation (RFCA) is effective in patients who tolerated VT well. They suggested that all inducible VTs should be treated with antiarrhythmic agents, ICD implantation, or RFCA, because they found a high rate of recurrent VT in patients with persistently inducible nonclinical VT. They achieved complete success in treating all inducible VTs, including nonclinical VTs in 11 of 35 patients. In our series, we attempted catheter ablation of inducible VTs in 8 patients as previously reported. ${ }^{24)}$ We achieved complete success in 1 patient and modified the cycle length of VT in another. The former patient died suddenly after 17 months and the latter developed recurrent, sustained VT 3 months after the procedure. The VTs of the other 6 patients could not be ablated. Because the patients with DCM have diffuse substrates for the reentrant circuit, it is difficult to completely treat all inducible VT by catheter ablation. In addition, because DCM is a progressive disease, VT with a new morphology may develop in the future. Therefore, catheter ablation seems to have limited value in the treatment of these patients.

Efficacy of class I agents vs amiodarone: The numbers of patients in the NCVT and CVT groups who had been treated with amiodarone did not differ significantly (5 patients vs 4 patients). Only 1 patient in the NCVT group and 2 in the CVT group who had been treated with amiodarone developed recurrent VT. In total, 10 of 15 patients $(67 \%)$ treated with class I agents experienced VT recurrence in contrast to 3 of 9 patients (33\%) given amiodarone. Although the difference was not significant, amiodarone seems to be more useful than class I agents 
in treating patients with life-threatening ventricular tachyarrhythmias in nonischemic dilated cardiomyopathy.

Study limitations: This study is not a prospective, randomized trial. Neither our programmed ventricular stimulation protocol nor the criteria used to define effective drug therapy based on EPS guided serial drug testing were changed during the study period. However, the long-term outcome might be influenced because fewer treatment options were available during the initial stages of the study since because this study included patients who had been admitted to the hospital in the early 1990s. Secondly, we could not always complete the programmed ventricular stimulation when clinical sustained VT was induced. If we performed more aggressive stimulation even after induction of CVT, NCVT might be increased. However, this would increase the risk of complications in such patients with severe left ventricular cardiomyopathy. In addition, we completed the PVS protocol if VT could not be induced under antiarrhythmic medication.

Conclusions: Induced nonclinical VT is as refractory to pharmacological therapy as induced clinical VT. In patients with nonclinical VT as well as clinical VT induced by programmed ventricular stimulation, EPS guided pharmacological therapy is limited and may require combination with ICD therapy. Amiodarone should decrease the recurrence rate of life-threatening ventricular tachyarrhythmia compared with class I agents in these patients.

\section{REFERENCES}

1. Huang SK, Messer JV, Denes P. Significance of ventricular tachycardia in idiopathic dilated cardiomyopathy. Observation in 35 patients. Am J Cardiol 1983; 51: 507-12.

2. Larsen L, Markham J, Haffajee CI. Sudden death in idiopathic dilated cardiomyopathy: Role of ventricular arrhythmias: PACE 1993; 16: 1051-9.

3. Tanabe T, Goto Y. Long-term prognostic assessment of ventricular tachycardia with respect to sudden death in patients with and without overt heart disease. Jpn Circ J 1989 Dec; 53 (12): 1557-64.

4. Kasanuki H, Ohnishi S, Tanaka E, Hirosawa K. Mechanism and prediction of sudden cardiac death in arrhythmia patients using electrophysiological studies. Jpn Circ J 1989 Dec; 53 (12): 1565-70.

5. Swerdlow CD, Winkle RA, Mason JW. Determinants of survival in patients with ventricular tachyarrhythmias. N Engl J Med 1983; 308: 1436-42.

6. Reddy CP, Sartini JC. Nonclinical polymorphic ventricular tachycardia induced by programmed cardiac stimulation: Incidence, mechanisms and clinical significance. Circulation 1980; 62: 988-95.

7. Constantin L, Martins JB, Kienzle MG, Brownstein SL, McCue ML, Hopson RC. Induced sustained ventricular tachycardia in nonischemic dilated cardiomyopathy: Dependence on clinical presentation and response to antiarrhythmic agents. PACE 1989; 12: 776-83.

8. Naccarelli GV, Fineberg NS, Zipes DP, Heger JJ, Duncan G, Prystowsky EN. Amiodarone: Risk factors for recurrence of symptomatic ventricular tachycardia identified at electrophysiologic study. J Am Coll Cardiol 1985; 6: 814-21.

9. Grimm W, Hoffmann J, Knop U, Winzenburg J, Menz V, Maisch B. Value of time- and frequency-domain analysis of signal-averaged electrocardiography for arrhythmia risk prediction in idiopathic dilated cardiomyopathy. PACE 1996; 19 [pt. II]: 1923-7. 
10. Buckingham TA, Thessen CC, Stevens LL, Redd RM, Kennedy HL. Effect of conduction defects on the signal-averaged electrocardiographic determination of late potentials. Am J Cardiol 1988; 61: 1265-71.

11. Gomes JA, Winters SL, Martinson M, Machac J, Stewart D, Targonski A. The prognostic significance of quantitative signal-averaged variables relative to clinical variables, site of myocardial infarction, ejection fraction and ventricular premature beats: a prospective study. J Am Coll Cardiol 1989; 13: 377-84.

12. Rothman SA, Hsia HH, Cossü SF, Chmielewski IL, Buxton AE, Miller JM. Radiofrequency catheter ablation of postinfarction ventricular tachycardia: Long-term success and the significance of inducible nonclinical arrhythmias. Circulation 1997; 96: 3499-508.

13. Kuller LH. Sudden death-definition and epidemiologic considerations. Prog Cardiovasc Dis 1980; 23: 1-12

14. Steel RGD, Torrie JH. Principles and Procedures of Statistics. New York, McGraw-Hill Book Co., Inc., 1960.

15. Buitleir M, Morady F, DiCarlo LA, Jr., Baerman JM, Krol RB. Immediate reproducibility of clinical and nonclinical forms of induced ventricular tachycardia. Am J Cardiol 1986; 58: $279-82$.

16. Morady F, DiCarlo LA, Jr., Baerman JM, Buitleir M. Comparison of coupling intervals that induce clinical and nonclinical forms of ventricular tachycardia during programmed stimulation. Am J Cardiol 1986; 57: 1269-73.

17. Buxton AE, Waxman HL, Marchlinski FE, Josephson ME. Electrophysiologic studies in nonsustained ventricular tachycardia: relation to underlying heart disease. Am J Cardiol 1983; 52: 985-91.

18. Horowitz LN, Josephson ME, Farshidi A, Spielman SR, Michelson EL, Greenspan AM. Recurrent sustained ventricular tachycardia 3. Role of the electrophysiologic study in selection of antiarrhythmic regimens. Circulation 1978; 56: 986-97.

19. Ruskin JN, DiMarco JP, Garan H. Out-of-hospital cardiac arrest: electrophysiologic observations and selection of long- term antiarrhythmic therapy. N Engl J Med 1980; 303: 607-13.

20. Rae AP, Spielman SR, Kutalek SP, Kay HR, Horowitz LN. Electrophysiologic assessment of antiarrhythmic drug efficacy for ventricular tachyarrhythmias associated with dilated cardiomyopathy. Am J Cardiol 1987; 59: 291-5.

21. Poll DS, Marchlinski FE, Buxton AE, Josephson ME. Usefulness of programmed stimulation in idiopathic dilated cardiomyopathy. Am J Cardiol 1986; 58: 992-7.

22. Chen X, Shenasa M, Borggrefe M, et al. Role of programmed ventricular stimulation in patients with idiopathic dilated cardiomyopathy and documented sustained ventricular tachyarrhythmias: inducibility and prognostic value in 102 patients. Eur Heart J 1994; 15: 76-82.

23. Milner PG, Dimarco JP, Lerman BB. Electrophysiological evaluation of sustained ventricular tachyarrhythmias in idiopathic dilated cardiomyopathy. PACE 1988; 11: 562-8.

24. Sato M, Sakurai M, Yotsukura A, et al. The efficacy of radiofrequency catheter ablation for the treatment of ventricular tachycardia associated with cardiomyopathy. Jpn Circ J 1997 Jan; 61 (1): 55-63. 